\title{
EFFET DES TOCOPHÉROLS DANS LA CONSERVATION DES BEURRES
}

\author{
par \\ O. COLAGRANDE et P. FONTANA \\ Instituts des Industries Agricoles et de Chimie agricole \\ Université Catholique du S. Cour, Piacenza (Italie)
}

On sait que les tocophérols sont considérés comme les responsables de la stabilité oxydative des huiles et des autres matières grasses [1-6].

Même dans les beurres a été trouvée une petite quantité de tocophérols [7-10].

Selon certains auteurs les beurres ayant une faible teneur en tocophérols ont une forte tendance à l'oxydation.

D'autres, au contraire, nient ou attribuent peu d'importance à la présence des tocophérols dans les beurres aux effets de l'autooxydation.

Swartling [11] a observé que l'addition de tocophérol en quantité variable (de 2,5 à $10 \mathrm{mg}$ p. 100) dans les beurres a une action pro-oxydante.

Reinart [12] a déterminé la teneur en tocophérols des beurres suédois. Il a observé que les valeurs maximales de 2-3 mg p. 100 se trouvent dans les beurres produits aux mois d'août, septembre, octobre, périodes pour lesquelles la rancidité oxydative est plus fréquente.

L'auteur retient, toutefois, que cet antioxydant est contenu dans les beurres en quantité trop petite pour pouvoir expliquer une action déterminante sur les phénomènes d'auto-oxydation.

Depuis longtemps nous nous sommes intéressés au problème de la détérioration du beurre conservé.

Dans un premier travail nous avons pu observer une intéressante relation entre le rancissement et la teneur en cuivre $d u$ beurre [13].

Dans cette recherche nous nous sommes proposés de certifier la présence des tocophérols dans des échantillons de beurre frais et d'une provenance sûre.

Nous nous sommes proposés aussi d'étudier l'effet de cet antioxydant à doses naturelles sur les phénomènes d'altération du beurre. 


\section{Matériaux et méthodes}

L'étude a été effectuée du printemps 1964 au printemps 1965 sur des échantillons de beurre prélevés tous les mois dans des beurreries de la province de Reggio Emilia.

Les beurres pris en examen étaient obtenus par écrémage spontané, et dans certaines beurreries on a pu prélever aussi des beurres obtenus par écrémage centrifuge.

Les échantillons prélevés au moment de la butyrification étaient mis dans un frigidaire portatif.

Sur tous les échantillons, le jour même du prélèvement, ont été faits les dosages suivants : matière grasse, cuivre total et soluble, acides polyinsaturés, tocophérols.

La teneur en tocophérols était dosée aveo la méthode de Erickson [14].

Les acides polyinsaturés ont été déterminés par la chromatographie en phase gazeuse [15] en utilisant un Pye argon chromatograph.

Pour les autres déterminations (matière grasse, cuivre total et soluble) nous avons appliqué les méthodes indiquées dans notre note précédente [13].

Le cuivre total était déterminé par colorimétrie (réaction par le diéthyldithiocarbamate).

Pour l'évaluation du cuivre soluble on procédait de cette façon : à $50 \mathrm{~g}$ de beurre fondu on ajoutait $50 \mathrm{~cm}^{3}$ d'eau bidistillée tiède $\left(40^{\circ} \mathrm{C}\right)$; agitait énergiquement l'émulsion pendant 5-6 minutes et on séparait ensuite la fraction aqueuse par centrifugation à 6000 tours/minute pendant 10 minutes. Afin d'être plus certain de la complète extraction du cuivre soluble, on répétait le lavage trois fois.

Les fractions aqueuses étaient ultrafiltrées sur cellophane.

Sur une fraction ultrafiltrée on déterminait le cuivre soluble par la réaction ci-dessus indiquée.

Des échantillons de beurre d'été choisis exprès étaient soumis à des épreuves d'auto-oxydation.

A ce sujet $50 \mathrm{~g}$ de chaque échantillon étaient mis dans des cuvettes de verre et conservés à l'obscurité pendant 48 heures en thermostat réglé à $30^{\circ} \mathrm{C}$.

La cinétique de l'oxydation était étudiée avec la mesure de l'indice de peroxyde faite après $1,4,8,24,48$ heures de conservation, en employant la méthode par nous indiquée [13].

A chaque prélèvement, les échantillons de beurre étaient mélangés à nouveau afin de favoriser l'oxydation de la masse.

On évaluait le comportement antioxygène des tocophérols par le temps nécessaire au développement de la rancidité oxydative. 


\section{Résultats et discussion}

Par les données des tableaux I, II, III on observe que la teneur en tocophérols des beurres examinés varie de 1,6 à $6 \mathrm{mg} \mathrm{p} .100$.

On remarque que les échantillons de beurre produits dans la période printemps-été sont plus riches en tocophérols en comparaison des échantillons de beurre d'hiver.

Cette variation est due à une plus grande richesse en tocophérols du fourrage vert.

Dans certains beurres de la même époque et de la même zone nous constatons quelques différences dans la teneur en tocophérols (tableau II). Ces différences peuvent être attribuées, à notre avis, à la technique employée dans la butyrification de la crème.

En effet, les données du tableau III font observer que les beurres de centrifuge produits dans la même période et dans la même zone sont plus pauvres en tocophérols par comparaison avec des beurres obtenus par crémage spontané.

On sait que la centrifugation provoque une perturbation de l'équilibre des composants de la membrane des globules gras du lait [16].

Les tocophérols qui sont absorbés sur cette membrane protectrice pourraient se détacher de la superficie des globules gras et passer dans le lait écrémé.

Cette hypothèse est validée par les données du tableau IV qui montrent que la teneur en tocophérols est plus élevée dans les crèmes spontanées que dans les crèmes de centrifuge obtenues par des mêmes laits.

Dans le tableau $V$ sont reportées les données relatives à l'influence des tocophérols sur les phénomènes d'oxydation.

Sur ce tableau les échantillons analysés ont été classifiés en quatre groupes.

Cette classification permet de comparer des groupes homogènes avec d'autres groupes qui différent les uns des autres par une seule variable.

Le premier groupe a en commun avec le second la quantité moyenne de cuivre soluble et la quantité d'acides polyinsaturés; il s'en différencie par la teneur en tocophérols.

Le troisième a en commun avec le second la valeur moyenne des tocophérols et d'acides polyinsaturés; il s'en différencie par la teneur en cuivre soluble.

Le quatrième groupe se différencie du troisième seulement par la teneur en acides polyinsaturés. 


\section{TABLEAU I}

ÉCHANTILLONS DE BEURRE D'HIVER (NOVEMBRE-MARS) PRÉLEVÉS DANS NEUF BEURRERIES (1)

\begin{tabular}{|c|c|c|c|c|}
\hline \multirow{2}{*}{$\begin{array}{c}\text { Matière } \\
\text { grasse } \\
\mathrm{g} \%\end{array}$} & \multirow{2}{*}{$\begin{array}{c}\text { Tocophérols } \\
\text { mg } \%\end{array}$} & \multicolumn{2}{|c|}{ Cuivre } & \multirow{2}{*}{$\begin{array}{c}\text { Acides } \\
\text { poly-insaturés } \\
\mathrm{g} \% \text { de m. } \mathrm{g} .\end{array}$} \\
\hline & & $\begin{array}{l}\text { Total } \\
\gamma / \mathrm{kg}\end{array}$ & $\begin{array}{l}\text { Soluble } \\
\text { r/kg }\end{array}$ & \\
\hline 84,1 & 1,60 & 45 & 10 & 1,45 \\
\hline 83,2 & 1,80 & 39 & 19 & 2,38 \\
\hline 82,7 & 1,38 & 63 & 28 & 1,62 \\
\hline 84,9 & 1,50 & 71 & 41 & 2,01 \\
\hline 84,4 & 2,01 & 100 & 58 & 1,49 \\
\hline 82,3 & 1,75 & 69 & 25 & 2,11 \\
\hline 85,8 & 1,39 & 101 & 43 & 2,24 \\
\hline 81,7 & 2,10 & 97 & 37 & 2,23 \\
\hline 83,7 & 1,98 & 83 & 18 & 2,92 \\
\hline
\end{tabular}

TABLEAU II

ECHANTILLONS DE BEURRE D'ÉTÉ (AVRIL-OCTOBRE) PRELEVES DANS NEUF BEURRERIES (1)

\begin{tabular}{|c|c|c|c|c|}
\hline \multirow{2}{*}{$\begin{array}{c}\text { Matière } \\
\text { grasse } \\
\text { g \% }\end{array}$} & \multirow{2}{*}{$\begin{array}{c}\text { Tocophérols } \\
\text { mg } \%\end{array}$} & \multicolumn{2}{|c|}{ Cuivre } & \multirow{2}{*}{$\begin{array}{l}\text { Acides } \\
\text { poly-insaturés } \\
\mathrm{g} \% \text { de m. g. }\end{array}$} \\
\hline & & $\begin{array}{l}\text { Total } \\
\gamma / \mathrm{kg}\end{array}$ & $\begin{array}{c}\text { Soluble } \\
\gamma / \mathrm{kg}\end{array}$ & \\
\hline 81,9 & 5,10 & 105 & 38 & 4,66 \\
\hline 83,2 & 4,70 & 211 & 83 & 3,12 \\
\hline 85,1 & 2,50 & 98 & 18 & 2,85 \\
\hline 82,7 & 2,51 & 73 & 33 & 3,35 \\
\hline 85,8 & 5,90 & 321 & 109 & 4,30 \\
\hline 84,3 & 2,49 & 89 & 15 & 3,81 \\
\hline 82,8 & 6,00 & 92 & 31 & 3,01 \\
\hline 81,7 & 4,80 & 96 & 42 & 4,94 \\
\hline 85,2 & 4,70 & 56 & 16 & 3,97 \\
\hline
\end{tabular}

(1) De chaque beurrerie on prélevait en moyenne trois échantillons. 
TABLEAU III

TENEURS EN TOCOPHÉROLS RELEVÉES DANS DES ÉCHANTILLONS DE BEURRE D'ÉTÉ PRÉLEVÉS DANS LES CINQ MÊMES BEURRERIES

\begin{tabular}{c|c}
\hline \hline & \\
$\begin{array}{c}\text { Beurres produits } \\
\text { de crèmes } \\
\text { spontanées }\end{array}$ & $\begin{array}{c}\text { Beurres produits } \\
\text { de crèmes } \\
\text { de centrifuge }\end{array}$ \\
Tocophérols & Tocophérols \\
mg \% & mg \% \\
& \\
& \\
4,80 & 3,04 \\
3,70 & 2,00 \\
3,47 & 1,93 \\
4,81 & 2,33 \\
3,32 & 1,59 \\
\hline
\end{tabular}

TABLEAU IV

TENEURS EN TOCOPHÉROLS RELEVÉES DANS CINQ ÉCHANTILLONS DE CREMMES SPONTANÉES ET DE CENTRIFUGE OBTENUS DES MÊMES LAITS

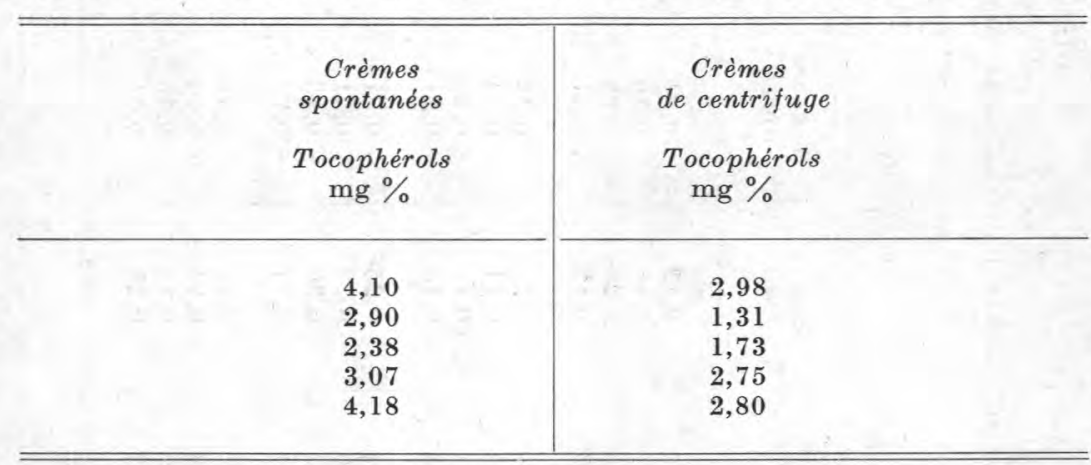

Par le tableau V et par le graphique (fig. 1) (où sont reportés, en abscisse, le temps en heures et, en ordonnée, les valeurs de l'indice de peroxyde) nous pouvons tout d'abord observer qu'après 24 heures de conservation dans les mêmes conditions expérimentales les valeurs de l'indice de peroxyde sont plus élevées dans les échantillons du $1^{\text {er }}$ groupe, qui sont plus pauvres en tocophérols en comparaison avec ceux du deuxième groupe.

En outre on observe qu'après 48 heures les valeurs de l'indice de peroxyde des deux groupes tendent à s'égaliser. 
TABLEAU V

VARIATION DE L'INDICE DE PÉROXYDE DANS SEIZE GCHANTILLONS DE BEURRE PENDANT L'AUTO-OXYDATION

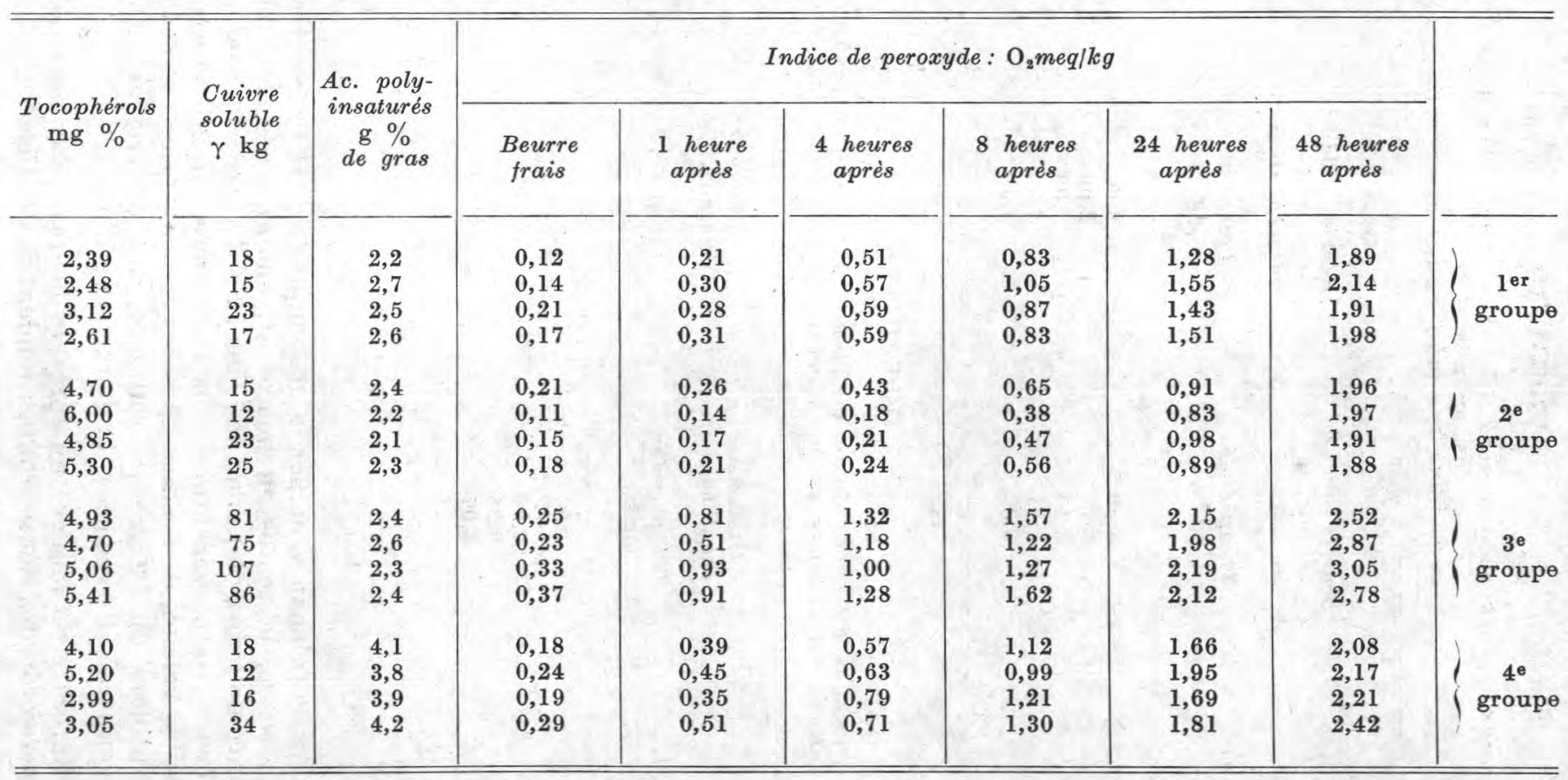


Ce phénomène serait du, à notre avis, aux tocophérols qui provoqueraient une prolongation de la période d'induction.

Les données du troisième groupe indiquent que cette action antioxygène des tocophérols est mitigée ou anéantie par la présence du cuivre soluble.

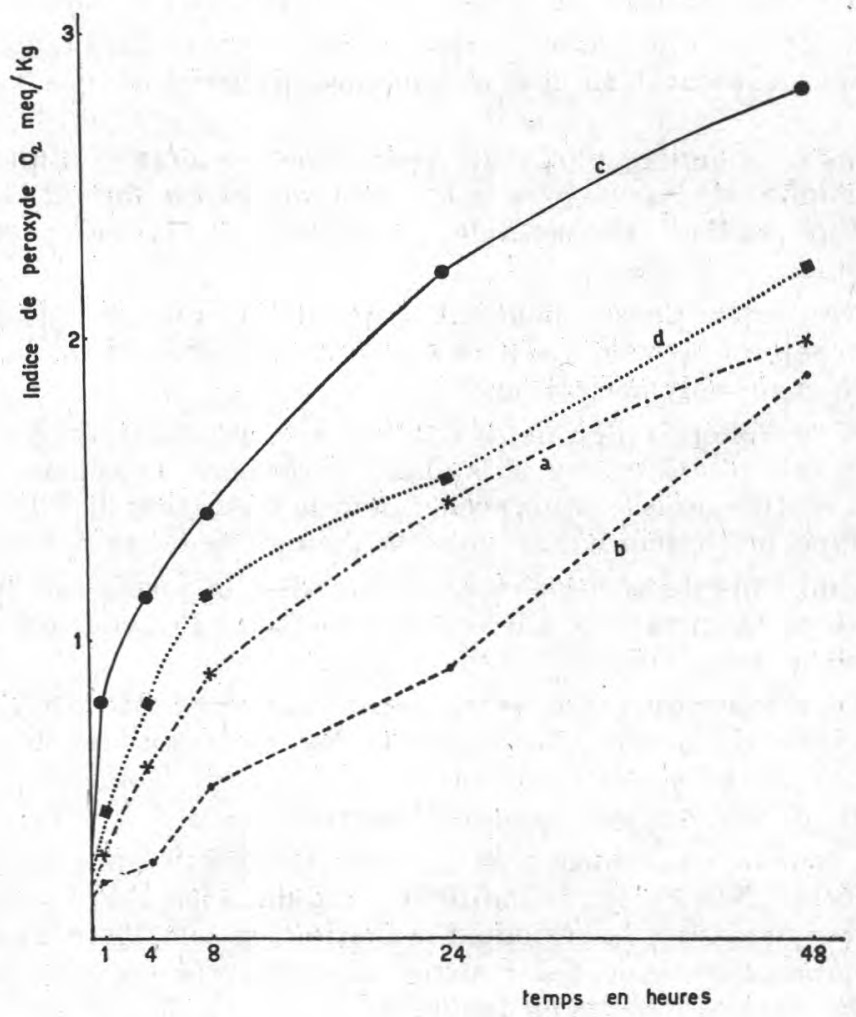

Fig. 1. - Cinétique d'oxydation des beurres à $30{ }^{\circ} \mathrm{C}$.

a) Échantillons de beurre ayant une teneur basse en tocophérols.

b) Échantillons de beurre ayant une teneur élevée en tocophérols.

c) Échantillons de beurre riche en cuivre soluble.

d) Échantillons de beurre riche en acides polyinsaturés.

Les beurres du $4^{\mathrm{e}}$ groupe, caractérisés par la présence de fortes doses d'acides polyinsaturés, se montrent moins stables à l'oxydation même s'ils contiennent des quantités relativement élevées de tocophérols et de petites quantités de cuivre soluble. 


\section{Conclusion}

Avant tout nous pouvons affirmer que les tocophérols exercent une action de protection oxydative sur les beurres pendant la période d'induction. Cette action peut être attribuée à la réaction des tocophérols avec les hydropéroxydes ou avec d'autres molécules actives qui se forment au début de l'auto-oxydation [18].

On sait, en effet, que l'auto-oxydation procède au moyen de réactions autocatalytiques auxquelles participent des radicaux libres.

Dans la première phase un hydrogène se détache d'un groupe méthylénique de l'acide gras et en conséquence on obtient la formation d'un radical responsable du début de la réaction autocatalytique

Après cette phase, pendant laquelle la quantité d'hydropéroxydes rejoint les valeurs élevées, prévalent les réactions de décomposition et de polymérisation.

Les tocophérols agiraient comme accepteurs d'oxygène dans la première phase et, en s'oxydant, fixeraient l'oxygène dissous dans la matière grasse et empêcheraient la libération de l'hydrogène du groupe méthylénique et donc la formation de radicaux libres.

Quand tous les tocophérols sont oxydés, la formation de radicaux libres ne trouve pas d'empêchements et l'oxydation peut se développer très vite.

Il faut remarquer que cette action antioxydante est liée à la composition du beurre. Par exemple, les beurres ayant une teneur élevée en tocophérols et en cuivre soluble sont moins stables que ceux ayant une teneur basse en cuivre.

La teneur en acides gras polyinsaturés influence l'effet des tocophérols. Nos épreuves indiquent que dans les beurres riches en acides polyinsaturés la période d'induction est sensiblement abrégée par comparaison avec des beurres plus pauvres en tels acides ot ayant les mêmes teneurs en tocophérols.

Cette observation pourrait expliquer la probable action prooxydante des tocophérols rapportée par certains auteurs [11].

En effet dans les beurres ayant une teneur élevée en acides polyinsaturés, la vélocité d'oxydation n'est pas pratiquement influencée par la petite quantité de tocophérols et elle se maintient toujours haute. Dans ces conditions, l'oxydation due aux acides polyinsaturés peut être attribuée par erreur aux tocophérols.

Ainsi, on constate que selon nos données les tocophérols agissent sur l'auto-oxydation du beurre en allongeant la période d'induction. Toutefois d'autres facteurs, tels que la teneur on acides polyinsaturés, la teneur en cuivre soluble etc., influencent cette action antioxygène jusqu'à l'anéantir. 


\section{Résumé}

Des épreuves d'auto-oxydation faites sur des échantillons de beurre frais, ont permis de prouver que les tocophérols contenus dans ces mêmes beurres avaient une action antioxydante en allongeant la période d'induction.

On a pu constater, en outre, que cette action antioxygène est mitigée ou anéantie par des facteurs inhérents à la composition du beurre.

\section{Summary}

In many experiments of auto-oxydation the tocopherols naturally contained in the butter were demonstrated to exert an antioxidative action in that they induce a longer period of induction; this activity can be furthermore counteracted or even nullified by certain butter chemical constituents as unsaturated fatty acids or copper soluble.

\section{RÉFÉRENCES BIBLIOGRAPHIQUES}

[1] Olcott (H. S.) and Emerson (O. H.). J. Am. Chem. Soc., 59, 1008, 1937.

[2] Hove (E. L.) and Hove (Z.), J. Biol. Chem., 156, 611 et 623, 1944.

[3] Grewahn (J.) and Daubert (B. F.). J. Am, Oil Chem. Soc., 25, 26, 1948.

[4] Quatre (M. L.). J. Biol. Chem., 175, 605, 1948.

[5] Lange (W.). J. Am. Oil Chem. Soc., 27, 414, 1950.

[6] Dugan (L. R.) jr and Kraybil (H. R.). J. Am. Oil Chem. Soc., 33, 527,1966

[7] QuAIFE (M. L.). J. Biol. Chem., 169, 513, 1947.

[8] Jens Nielsen (J.), Fisker (A. N.), Pederesen (A. H.), Prange (I.). Sondergaard (E.) and Dam (H.). J. Dairy Res., 20, 333, 1953.

[9] Harris (P. L.), Quaife (M. L.) and Swanson (W. J.). J. Nutr., 40, 367, 1950.

[10] Wевв (B. H.) and JoHnson (A. H.). Fundamentals of dairy chemistry, The Avi Publishing Company, Inc. 1965.

[11] Swarthing (P.). Proc. 12 Intern. Dairy Congr., (Stockholm) 2, 375, 1949.

[12] Reinart (A.). Proc. 12 Intern. Dairy Congr., (Stockholm), 2, 405, 1949

[13] Fontana (P.) et Colagrande (O.). Il Latte, 37, 960, 1963.

[14] Eriokson (D. R.) and Dunkley (W. L.). Anal. Chem., 36, 1055, 1964.

[15] Méthodes officielles d'analyses des huiles et des gras, Suppl. I, Institute Polyg. de l'État. Rome, 1963.

[16] Colagrande (O.) et Fontana (P.). XVII Congrès Intern. de Laiterie, B : $2,227,1966$.

[17] Erickson (D. R.), Dunkley (W. L.) and Smith (L. M.). J. Food Sci., $29,269,1964$.

[18] Khan (N. A.), Oléagineux, 13, 331, 1958. 